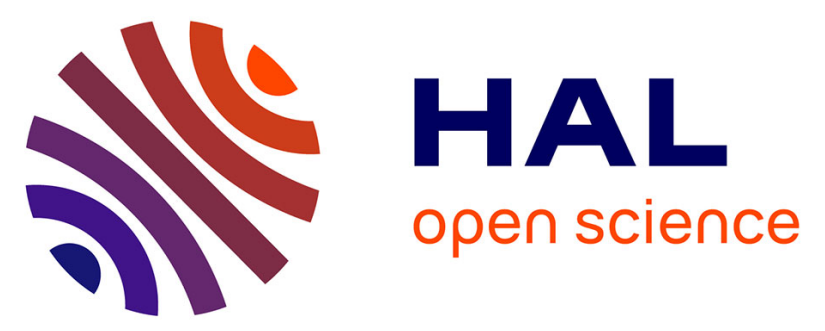

\title{
Boosting Hot Electron-Driven Photocatalysis through Anisotropic Plasmonic Nanoparticles with Hot Spots in Au- TiO 2 Nanoarchitectures
}

\author{
Ana Sousa-Castillo, Miguel Comesaña-Hermo, Benito Rodriguez-Gonzalez, \\ Moisés Pérez-Lorenzo, Zhiming Wang, Xiang-Tian Kong, Alexander Govorov, \\ Miguel A Correa-Duarte
}

\section{To cite this version:}

Ana Sousa-Castillo, Miguel Comesaña-Hermo, Benito Rodriguez-Gonzalez, Moisés Pérez-Lorenzo, Zhiming Wang, et al.. Boosting Hot Electron-Driven Photocatalysis through Anisotropic Plasmonic Nanoparticles with Hot Spots in Au- TiO 2 Nanoarchitectures. Journal of Physical Chemistry C, 2016, 120 (21), pp.11690-11699. 10.1021/acs.jpcc.6b02370 . hal-02570512

\section{HAL Id: hal-02570512 \\ https://hal.science/hal-02570512}

Submitted on 12 May 2020

HAL is a multi-disciplinary open access archive for the deposit and dissemination of scientific research documents, whether they are published or not. The documents may come from teaching and research institutions in France or abroad, or from public or private research centers.
L'archive ouverte pluridisciplinaire $\mathbf{H A L}$, est destinée au dépôt et à la diffusion de documents scientifiques de niveau recherche, publiés ou non, émanant des établissements d'enseignement et de recherche français ou étrangers, des laboratoires publics ou privés. 


\section{Boosting Hot Electron-Driven Photocatalysis through}

\section{Anisotropic Plasmonic Nanoparticles with Hot Spots in Au-}

\section{$\mathrm{TiO}_{2}$ Nanoarchitectures}

Ana Sousa-Castillo, ${ }^{\dagger}$ Miguel Comesaña-Hermo,${ }^{\dagger}$ Benito Rodríguez-González, ${ }^{\dagger}$ Moisés Pérez-Lorenzo, ${ }^{\dagger}$ Zhiming Wang, ${ }^{\ddagger}$ Xiang-Tian Kong, ${ }^{\ddagger}, \S$ Alexander O. Govorov, ${ }^{*}$, and Miguel A. Correa-Duarte ${ }^{*, \dagger}$

†Department of Physical Chemistry, Biomedical Research Center (CINBIO), and Institute of Biomedical Research of Ourense-Pontevedra-Vigo (IBI), Universidade de Vigo, 36310 Vigo, Spain

Institute of Fundamental and Frontier Sciences, University of Electronic Science and Technology of China, Chengdu 610054, PR China; State Key Laboratory of Electronic Thin Films and Integrated Devices, University of Electronic Science and Technology of China, Chengdu 610054, PR China

${ }^{\S}$ Department of Physics and Astronomy, Ohio University, Athens, Ohio 45701, United States 


\section{ABSTRACT}

The use of plasmonic metal nanoparticles as photosensitizers has undergone a strong development in the last few years given their ability to increase the activity of semiconductors into the visible and near infrared regions. The present work reports an experimental and theoretical study on the critical influence that shape anisotropy of gold nanoparticles exerts on the photocatalytic performance of $\mathrm{Au}-\mathrm{TiO}_{2}$ nanoarchitectures. The obtained results show that for a given amount of metallic material, Au nanostars endow titania with a strongly-enhanced catalytic efficiency compared to that found in the presence of Au nanospheres or nanorods. This is ascribed to the ability of nanostars to locally create extremely large electromagnetic field enhancements around their spikes, which ensures an increased population of hot electrons close to the interface between the metal and the semiconductor. Therefore, these nanostructures exhibit a novel regime of photocatalytic activity that could be described as plasmonic hot-spot photocatalysis. Numerical simulations confirm that the hot electron injection is a feasible mechanism behind the photosensitization process and that the nanostars should have the strongest photochemical response. These results pave the way for a more rational design of the plasmonic component in the search for high-performance photocatalytic nanoreactors operating under visible and NIR light. 
Titanium dioxide is a robust, nontoxic and inexpensive material that presents outstanding photocatalytic and photoelectrochemical properties. ${ }^{1}$ Taking advantage of these features, $\mathrm{TiO}_{2}$ nanoparticles have been used in the last decades in a broad variety of fields, ranging from photovoltaics or water treatment to photocatalytic water splitting. ${ }^{2}$ Nevertheless, as a wide band gap semiconductor $(3.2 \mathrm{eV}) \mathrm{TiO}_{2}$ can only absorb UV photons. Such a limitation narrows these remarkable properties to a segment of the solar spectrum that only accounts for $5 \%$ of the total radiation. For that reason, different methodologies have been developed in the last years in order to extend the activity of this semiconductor to the visible and near-IR regions. ${ }^{3-5}$ Among these approaches, the use of plasmonic metal nanoparticles as photosensitizers constitutes a promising strategy due to the ability of these particles to harvest visible light and transfer that energy to the semiconductor, enhancing charge separation in $\mathrm{TiO}_{2}{ }^{6,7} \mathrm{In}$ this regard, two are the mechanisms that allow plasmonic sensitization to take place: plasmon induced resonant energy transfer (PIRET) and direct electron transfer (DET). PIRET consists in the non-radiative dipoledipole coupling between the plasmon of the metal and the electron-hole pairs in the semiconductor. ${ }^{8-11}$ In this case, plasmonic photocatalysis can proceed even in the presence of an insulating barrier between the metal and the semiconductor. DET, also referred to as hot electron injection, involves the transfer of plasmonic electrons over the interfacial Schottky barrier established between the Fermi level of the metal and the conduction band of the semiconductor. ${ }^{12-14}$ For this mechanism, a proper band alignment and electronic coupling between the metal and the semiconductor are required. As a result, an intimate contact between both components becomes necessary. For a given photocatalytic system, both PIRET and DET may coexist or alternatively, being only one responsible for the performance of the metal-semiconductor system. In any case, the absorption spectra of the metal and the semiconductor together with the structural configuration of the photocatalytic system will have a critical influence on the photocatalytic activity. In particular, plasmonic hot spots can play a role as centers of active generation of energetic electrons for the injection process. ${ }^{8,15-17}$

When it comes to plasmonic photosensitization of titania, Au is frequently preferred over other metals that also exhibit absorption in the visible region such as $\mathrm{Ag}$ or $\mathrm{Cu}$. This is due to its lower tendency to oxidation and higher robustness which in the end, ensures the necessary long-term photochemical stability of the composite. ${ }^{18}$ In this case, the absence of spectral overlapping between the plasmon band of gold and the absorption band of $\mathrm{TiO}_{2}$ 
allows ruling out a PIRET mechanism in favor of a process exclusively driven by hot electron injection. It must be also noted that the plasmon band is extremely sensitive to the size and shape of gold nanoparticles (AuNPs). ${ }^{19}$ Therefore, variations in these morphological features can be used to induce changes in the intrinsic absorption signatures of the particles, thus enhancing the catalytic efficiency of $\mathrm{Au}-\mathrm{TiO}_{2}$ nanocomposites. Along these lines, tuning the size of plasmonic AuNPs has been shown to lead to remarkable differences in terms of photocatalytic performance. ${ }^{20-22}$ Nevertheless, the conclusions drawn in these works significantly differ from one to another. This can be attributed to the particular synthetic methodologies used in each case and hence, the distinct semiconductor/metal interface of the resulting composites. In the end, these structural factors will affect the efficiency of plasmonic photosensitization of titania. ${ }^{7}$

Regarding the shape, only few reports have tackled the role played by the anisotropy of AuNPs on the photocatalytic activity of $\mathrm{Au}-\mathrm{TiO}_{2}$ architectures. Li et al. have introduced three different aspect ratio (AR) Au nanorods (AuNRs) into porous $\mathrm{TiO}_{2}$ shells observing that the catalytic efficiency of these composites is altered as the rod AR is increased. ${ }^{23}$ In this case, the AuNR core with an intermediate AR value exhibits a greater catalytic effect for the oxidation of benzyl alcohol than that observed in the presence of those with a higher and lower aspect ratio. ${ }^{23}$ In this respect, it is claimed that while an increased AR favors the adsorption of the reactants thus improving the oxidation, a further rise in this value shifts the plasmon band to a longer wavelength region with a lower photon energy which results in the decrease of the photocatalytic performance. As a result, a medium AR is found to meet the necessary balance between both factors. Hu et al. have synthesized AuNPs (spheres and rods) onto ultrathin $\mathrm{TiO}_{2}$ nanosheets for the photocatalytic degradation of rhodamine $\mathrm{B}(\mathrm{RhB}){ }^{24}$ The authors obtained faster degradation rates in the presence of rods than that observed when using Au nanospheres. This is ascribed to the broader plasmon band of AuNRs which enables to harvest a wide range of visible and NIR light, thus enhancing the photocatalytic performance. Similar results were obtained when other morphologies with relatively broad absorption bands were compared with spherical nanoparticles. ${ }^{25}$

Despite the vast range of scientific contributions in this field, a complete understanding of the mechanisms governing the catalytic performance of $\mathrm{Au}-\mathrm{TiO}_{2}$ architectures remains elusive. For this reason, a comprehensive 
experimental and theoretical analysis of the different features affecting the photocatalytic properties of these nanocomposites is developed herein. In this case, particular attention has been paid to the influence of nanoparticle anisotropy. With this aim, Au nanostars have been used as photosensitizers given their ability to locally create extremely large electromagnetic field enhancements in their spikes (hot-spots), ${ }^{26}$ which ensures an increased population of hot electrons close to the interface between the metal and the semiconductor. Moreover, the catalytic behavior of AuNSTs has been compared to that found in the presence of AuNPs of different sizes and shapes in order to elucidate the impact that their corresponding electromagnetic field enhancements may exert on the performance of $\mathrm{Au}-\mathrm{TiO}_{2}$ nanocomposites.

\section{RESULTS AND DISCUSSION}

For comparison purposes, sub-micrometric silica beads have been used as supports for the adsorption of all the $\mathrm{Au}$ and $\mathrm{TiO}_{2}$ nanoparticles employed in this study. This synthetic approach not only provides a remarkable colloidal stability to the system but also a high degree of control over the deposition of the metal and the semiconductor materials onto the support. Along these lines, a highly homogeneous distribution of both species onto the surface of the beads is achieved and thus, a reproducible $\mathrm{Au}-\mathrm{TiO}_{2}$ interface throughout all the samples can be attained. The good reproducibility of the interface together with a tight control of the reaction conditions (concentrations, temperature, irradiation time, etc.) allow for a careful evaluation of the role played by the morphological aspects of plasmonic nanoparticles in these hybrid architectures. Furthermore, numerical simulations of the electron injection mechanism from $\mathrm{Au}$ to $\mathrm{TiO}_{2}$ have been carried out in order to verify that the obtained experimental results can be related to an electron transfer mechanism between these two materials. 
a
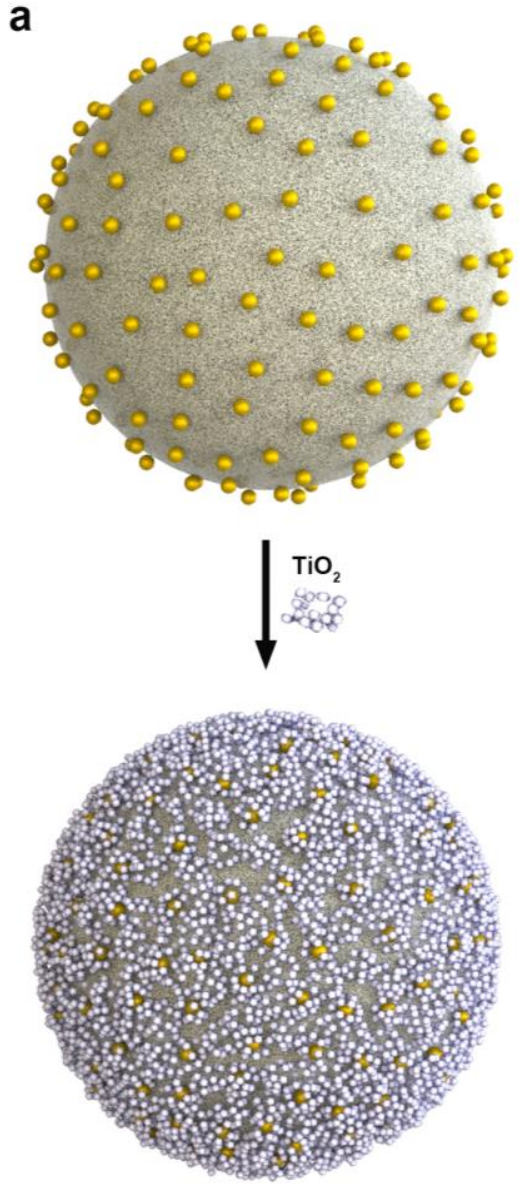

b
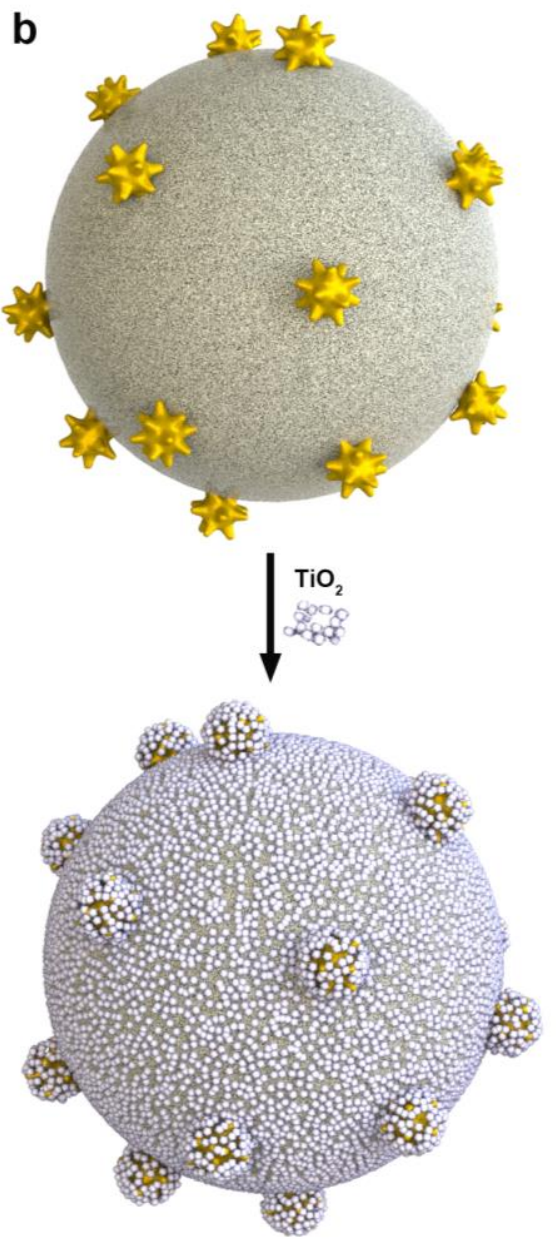

C
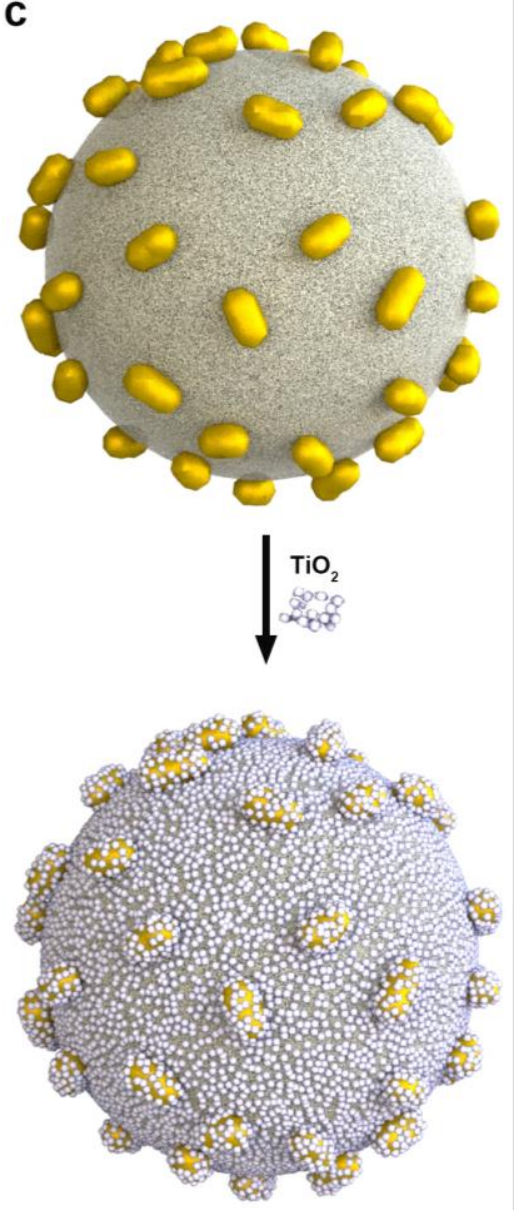

Scheme 1. Illustration depicting the deposition of $\mathrm{TiO}_{2}$ nanoparticles onto (a) $\mathrm{SiO}_{2} @ \mathrm{Au}$ nanospheres; (b) $\mathrm{SiO}_{2} @ \mathrm{Au}$ nanostars, and (c) $\mathrm{SiO}_{2} @ \mathrm{Au}$ nanorods.

The route for the fabrication of the photocatalysts is partially depicted in Scheme 1 (see Experimental Methods for further details). This protocol comprises the preparation of monodisperse silica beads by the Stöber method, ${ }^{27}$ and their subsequent functionalization with a positively charged monolayer of polyelectrolyte (poly(allylamine hydrochloride), PAH). After that, the controlled deposition of differently shaped gold nanoparticles (Figure 1ac) onto the surface of the $\mathrm{SiO}_{2} @ \mathrm{PAH}$ composites is carried out. Then, a second layer of PAH is adsorbed onto the $\mathrm{SiO}_{2} @ \mathrm{PAH} @$ AuNP nanostructures in order to ensure the electrostatic binding of the negatively charged $\mathrm{TiO}_{2}$ nanoparticles in the last step of the procedure. In this regard, anatase $\mathrm{TiO}_{2}(\geq 99 \%)$ has been used as semiconductor material given its enhanced photocatalytic activity when compared with other $\mathrm{TiO}_{2}$ phases. ${ }^{28,29}$ After the deposition of $\mathrm{TiO}_{2}$, a multilayered $\mathrm{SiO}_{2} @ \mathrm{AuNPs@} @ \mathrm{TiO}_{2}$ composite is obtained. 


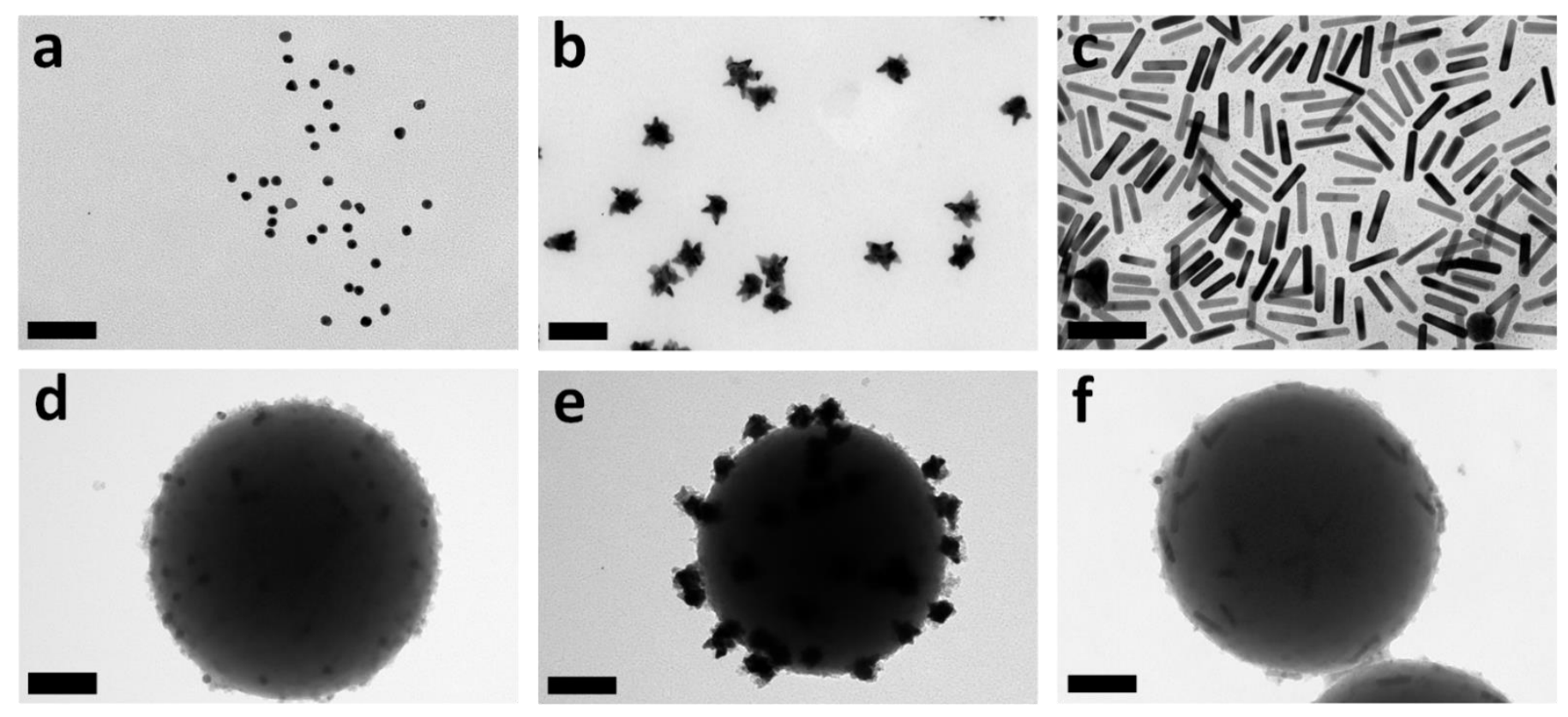

Figure 1. TEM images of (a-c) different-shaped AuNPs and (d-f) $\mathrm{SiO}_{2} @ \mathrm{AuNP} @ \mathrm{TiO}_{2}$ nanocomposites. From left to right: nanospheres, nanostars, and nanorods. Scale bars: $100 \mathrm{~nm}$.

The morphological analysis of these nanocomposites has been carried out by transmission electron microscopy (TEM) (Figure 1e-f). These images confirm the immobilization and homogeneous distribution of $\mathrm{TiO}_{2}$ onto the silica beads while the different AuNPs are kept individually sandwiched between both oxides (Figure S1). Likewise, these nanocomposites are found to exhibit high stability and dispersibility in solution (Figure S1-4). In the same line, STEM elemental mapping through XEDS analysis shows that the AuNPs (in this case, Au nanorods) retain their structural integrity throughout the assembly process (Figure S4-5). Furthermore, HRTEM images confirm the crystalline nature of the $\mathrm{TiO}_{2}$ layer deposited onto the AuNPs and the existence of a continuous interface between both components (Figure S6). The good recovery capabilities of these composites also suggest a good recycling potential of these photocatalysts.

The important differences in the absorption signatures of the three different morphologies chosen for this study (nanospheres (AuNSs), nanostars (AuNSTs) and nanorods (AuNRs)) can be clearly observed in Figure 2a. AuNSs with a diameter of $14 \mathrm{~nm}$ present a single and narrow absorption band with a maximum centered at $520 \mathrm{~nm}$ while the anisotropic particles exhibit more complex fingerprints. Even though the shape of their absorption spectra is different from each other, both anisotropic objects (AuNRs and AuNSTs) show two plasmon bands. In the case 
of AuNRs, the two well-separated absorption bands correspond to the transverse $(\sim 520 \mathrm{~nm})$ and longitudinal $(925 \mathrm{~nm})$ plasmon modes of these nanoparticles. For AuNSTs, the two different bands observed match the core (shoulder at $550 \mathrm{~nm}$ ) and outer spikes (broad band centered at $710 \mathrm{~nm}$ ) typical of these nanostructures. On the other hand, the UV-visible spectra of the corresponding composites display a strong absorption band at low wavelengths which is not present in the spectra of the free plasmonic objects in solution (Figure 2b). Such an effect arises as a consequence of the high absorption of $\mathrm{TiO}_{2}$ in the UV region together with an important scattering contribution of the $\mathrm{SiO}_{2}$ beads. Nevertheless, the LSPR signatures of the different Au morphologies are still discernible and most importantly, their absorption maxima show no noticeable shift, as observed in the case of the broad band of AuNSTs covering most of the visible region (blue line in Figure 2b). As aforementioned, the distinctive absorption signatures of these composites are expected to play a crucial role on their photocatalytic activity due to the different electromagnetic field enhancements associated to each of them.

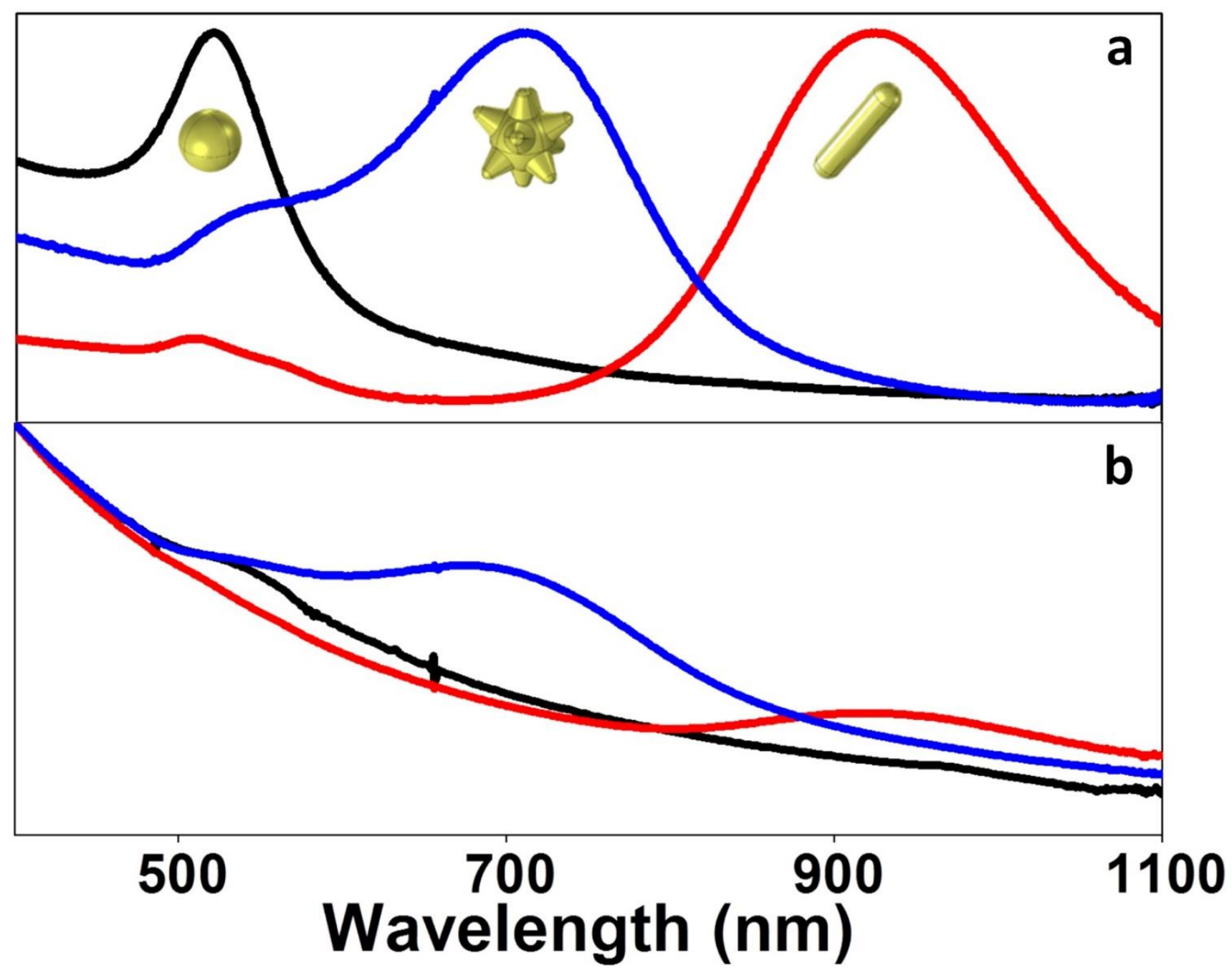


Figure 2. (a) UV-Vis-NIR spectra of free AuNPs in solution: nanospheres (black), nanostars (blue) and nanorods (red); (b) UV-Vis-NIR spectra of AuNPs adsorbed onto $\mathrm{SiO}_{2}$ beads after coating with $\mathrm{TiO}_{2}$ nanoparticles:

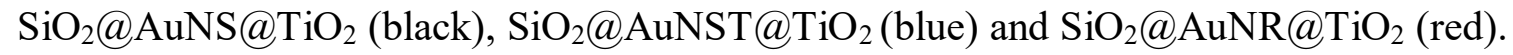

At this stage, the photocatalytic efficiency of each composite has been evaluated through the sunlight-mediated degradation of Rhodamine B, an archetypical and particularly harmful industrial dye. Thus, photo-oxidation of $\mathrm{RhB}$ in the presence of the $\mathrm{SiO}_{2} @ \mathrm{Au} @ \mathrm{TiO}_{2}$ architectures has been monitored by following the decrease in the absorption maximum of this organic compound $(554 \mathrm{~nm})$ under irradiation from a solar simulator covering a spectral range from 350 to $2400 \mathrm{~nm}$ (Figure 3). In this way, most of the UV contribution is eliminated and thus, the degree of photosensitization of $\mathrm{TiO}_{2}$ can be more carefully assessed.
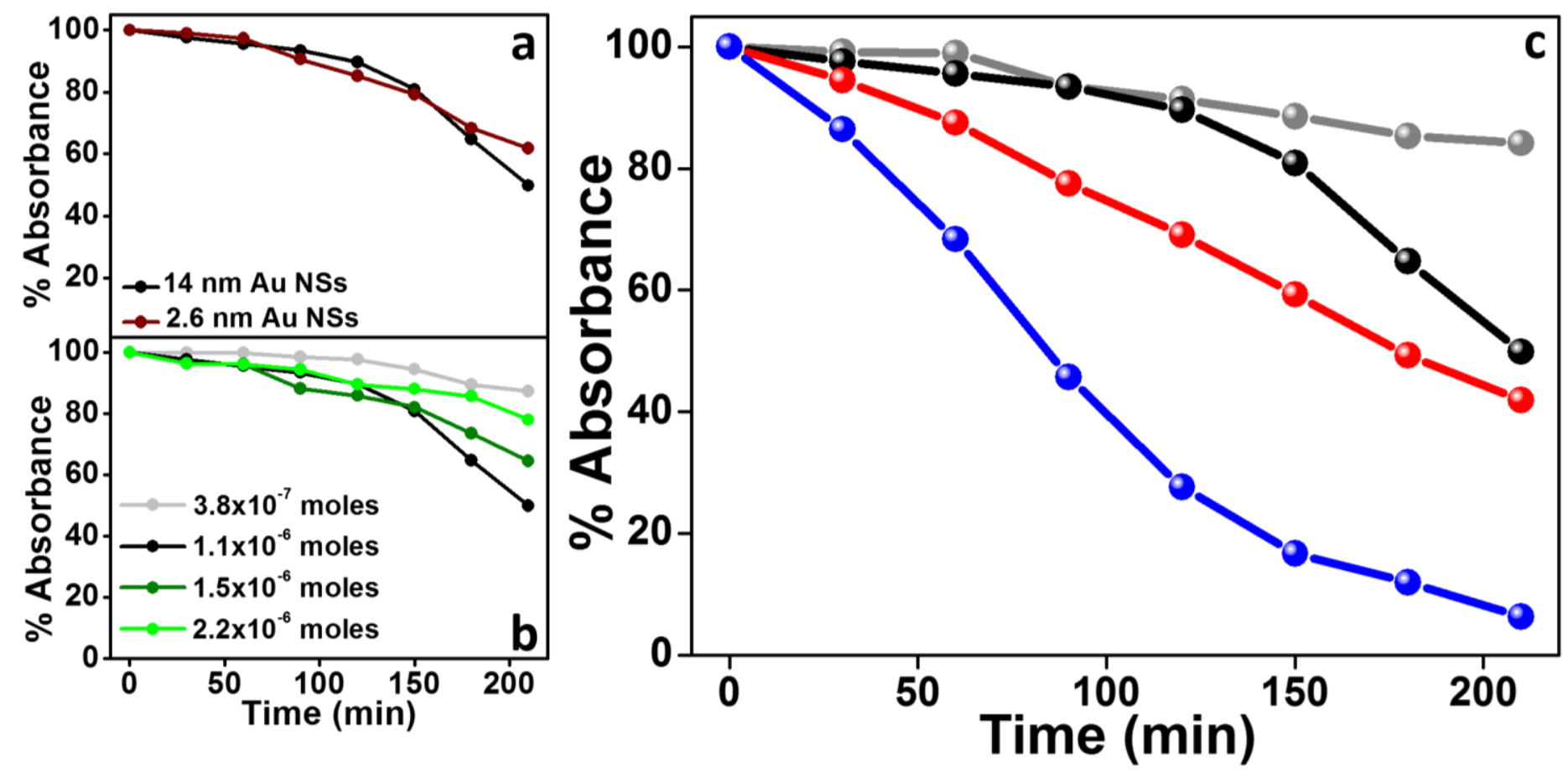

Figure 3. Photodegradation rate of $\mathrm{RhB}$ in the presence of (a) different-sized Au nanospheres, (b) different amounts of $14 \mathrm{~nm} \mathrm{Au}$ nanospheres (moles of metallic $\mathrm{Au}$ in the sample), and (c) different-shaped AuNPs: $\mathrm{SiO}_{2} @ \mathrm{TiO}_{2}$ as control (grey), $\mathrm{SiO}_{2} @ \mathrm{AuNS} @ \mathrm{TiO}_{2}$ (black), SiO $2 @ \mathrm{AuNR} @ \mathrm{TiO}_{2}$ (red) and $\mathrm{SiO}_{2} @ \mathrm{AuNST}_{2} \mathrm{TiO}$ (blue). 
In a first set of experiments, the effect of the Au nanoparticle size on the photocatalytic activity of the composites has been evaluated. Previous studies differ in their final conclusions regarding this subject. Some authors have reported a value of $8.6 \mathrm{~nm}$ as the size leading to the highest catalytic efficiency, while others place this mark between 12 and $15 \mathrm{~nm}^{20-22}$ In the present work, a decrease in the size of AuNSs from 14 to $2.6 \mathrm{~nm}$ induces a slight decrease in the photocatalytic activity of the $\mathrm{SiO}_{2} @ \mathrm{AuNS} @ \mathrm{TiO}_{2}$ composites (Figure 3a). This effect can be attributed to the absence of SPR for nanoparticles smaller than $3 \mathrm{~nm}$, which stresses the pivotal importance of the optical properties of AuNPs as photosensitizing agents.

Metal nanoparticle dosage constitutes another factor to take into consideration when investigating photocatalytic performance. In this study, an initial increase in the amount of gold (for a given concentration of $\mathrm{SiO}_{2}$ and $\mathrm{TiO}_{2}$ ) leads to a faster degradation of $\mathrm{RhB}$. However, when exceeding a certain threshold a further rise in the amount of photosensitizer is accompanied by a substantial decrease in the catalytic efficiency of the composites (Figures $3 \mathrm{~b}$ and S7). This inhibitory effect has been previously reported by other authors and is associated to the annihilation of electron-hole pairs under specific Au concentrations. In this case, metal nanoparticles behave as recombination centers resulting in the loss of the necessary reactive species that contribute to the photocatalytic reaction. ${ }^{30}$ The influence that the so-called "screening effect" ${ }^{30}$ may have on these results can easily be ruled out considering that Au nanoparticles are deposited below $\mathrm{TiO}_{2}$, thus facilitating the access of photons to the semiconductor.

After adjusting the Au dosage leading to the optimal photosensitizing behavior, the role played by the metal nanoparticle shape on the photocatalytic activity of $\mathrm{Au}-\mathrm{TiO}_{2}$ nanoarchitectures has been carefully examined (Figure 3c). As a control experiment, photodegradation of $\mathrm{RhB}$ has been carried out in the presence of $\mathrm{SiO}_{2} @ \mathrm{TiO}_{2}$ composites. Given the lack of AuNPs, only a limited fraction of dye (16\%) is oxidized after an irradiation time of more than $3 \mathrm{~h}$. The observed conversion can be attributed to the narrow interval of UV wavelengths (350-400 $\mathrm{nm}$ ) utilized by the solar simulator, which provides the necessary high energy photons to excite $\mathrm{TiO}_{2}$ in the absence of photosensitizer. Conversely, an important degree of photo-oxidation is observed in the presence of AuNPs after the same period of time. In this case, $14 \mathrm{~nm}$ AuNSs allow for a $50 \%$ decomposition of RhB while AuNRs display a slightly improved efficiency (59\%) after 210 min. Notably, an almost quantitative degradation 
of RhB is observed when using AuNSTs as photosensitizing material (94\%). By far, this excellent conversion makes Au nanostars the best photosensitizer of all the different-shaped metal nanoparticles synthesized herein. This superior performance can be ascribed to the extremely large enhancement of the electromagnetic field around the spikes of AuNSTs, leading to the formation of the so-called hot-spots. ${ }^{26}$ Such an enhancement leads to a more effective injection of hot electrons across the Schottky barrier to the conduction band of $\mathrm{TiO}_{2}$, thus triggering the creation of excitons and the subsequent formation of the necessary radical species that participate in the decomposition of RhB. Interestingly, composites synthesized with Au nanorods with smaller aspect ratio (62 nm x $20 \mathrm{~nm}$ ) whose main absorption signature overlaps that of the Au nanostars used in this study, present a significantly lower photocatalytic efficiency than that presented by the AuNSTs (Figure 4a,b). Such result highlights the importance of the hot-spots created at the spikes of the AuNSTs as the main reason for the formation of hot electrons that trigger the enhanced photocatalytic activity observed in these architectures. Even though the intensity of the local fields produced by the surface plasmons decay exponentially with the distance, the intimate contact between the metal and the semiconductor allows an efficient interaction between both components. Furthermore, the strong scattering of these AuNSTs may also lead to an improved optical concentration and thus, an enhanced light absorption of $\mathrm{TiO}_{2}{ }^{31}$

At this point, it is also important to exclude a direct photosensitization of $\mathrm{TiO}_{2}$ by $\mathrm{RhB}$ as a plausible mechanism that could compete with the plasmonic hot-electron photosensitization as responsible for the photodegradation of the organic molecule. ${ }^{8,32}$ To study this issue a glass colored filter that blocks all UV and visible contribution of the irradiation profile, and hence all photons of the excitation source with $\lambda<700 \mathrm{~nm}$, has been introduced in the experimental setup, excluding therefore a direct excitation of the dye (Figure 4c). In this manner, $\mathrm{SiO}_{2} @ \mathrm{Au} @ \mathrm{TiO}_{2}$ composites where the plasmonic component comprises long AuNRs $(105 \times 16 \mathrm{~nm})$ have been chosen as photosensitizers. Herein, the longitudinal plasmon band of the AuNRs is shifted deeper into the NIR region of the electromagnetic spectrum (maximum at $1102 \mathrm{~nm}$ ), being therefore unaffected by the suppression of photons at UV and visible frequencies. Interestingly, the photocatalytic capabilities of these nanostructures have been proven to be the same, independently of the presence or absence of the filter (Figure 4d), confirming therefore that the main mechanism behind the photodegradation of the dye resides in the electron transfer between 
the plasmonic component and the $\mathrm{TiO}_{2} \mathrm{NPs}$. At the same time, the lower degradation of the dye with respect to nanocomposites holding shorter AuNRs (35\% instead of the previous $59 \%$ of degradation) can be explained as a consequence of the large shift of the main absorption band to longer wavelengths. This displacement leads to the excitation of an important population of conduction electrons that do not have, when excited, enough energy to be transferred to the conduction band of the semiconductor (vide infra).
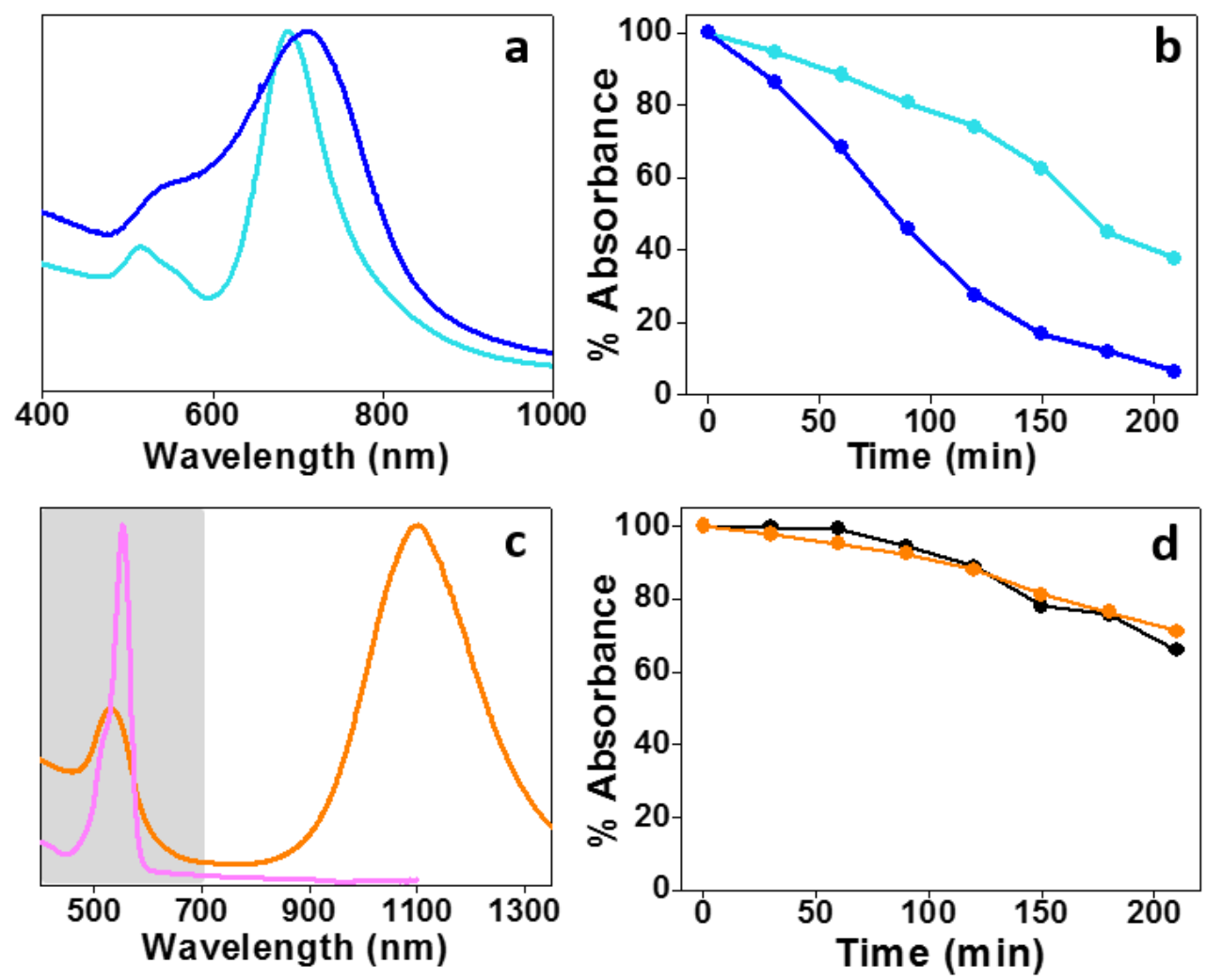

Figure 4. (a) UV-Vis-NIR spectra of the short AuNRs (cyan) and the AuNSTs (blue), with (b) their respective degradation profiles when forming part of the $\mathrm{SiO}_{2} @ \mathrm{Au} @ \mathrm{TiO}_{2}$ nanocomposites. (c) UV-Vis-NIR spectrum of the long AuNRs (orange), with a shadowed region that represents the portion of electromagnetic spectrum that has been blocked in the solar simulator with a colored glass filter $(<700 \mathrm{~nm})$. The absorption spectrum of RhB (pink) is shown for comparison purposes. (d) Degradation rates of the $\mathrm{SiO}_{2} @$ long AuNRs@TiO 2 composites with (orange) and without (black) the filter.

The experimental reaction rates for $\mathrm{RhB}$ degradation can be modeled for the different morphologies in terms of hot electron injection between the Fermi level of the metal nanoparticle and the conduction band of the 
semiconductor. In this direction, the theoretical model developed by Govorov and coworkers ${ }^{16,33}$ on the photogeneration of hot plasmonic carriers in metal nanoparticles can be applied to simulate the kinetics of electron photo-injection through a Schottky barrier. These works highlight that, under optical excitation, the Fermi sea of electrons in a metal nanoparticle acquires non-equilibrium electrons (shown in red in the energy diagram of Figure 5b). According to the quantum theories, ${ }^{16,34,35}$ the distribution of these non-equilibrium electrons exhibits two characteristic intervals: (1) the low-energy excitation region near the Fermi level and (2) the high-energy excitation contribution, which is flat and extends over the interval $\mathrm{E}_{\mathrm{F}}<\mathrm{E}<\mathrm{E}_{\mathrm{F}}+\hbar \omega$. This formalism has been recently used for the description of several hot electron experiments. ${ }^{17,36}$ In this model the barrier between the plasmonic nanoparticle and the semiconductor is denoted as $\Delta \mathrm{E}_{\text {barrier }}$ (Figure 5), whose value at an interface between the Fermi energy of $\mathrm{Au}$ and the conduction band of $\mathrm{TiO}_{2}$ is $\Delta \mathrm{E}_{\text {barrier }} \approx 1 \mathrm{eV}$. Such a value corresponds to an optical wavelength of $1240 \mathrm{~nm}$, meaning that photons with $\lambda<1240 \mathrm{~nm}$ are able to create excited electrons with enough energy to overcome this energy barrier, thus being able to be injected in the conduction band of $\mathrm{TiO}_{2}$ nanoparticles. Since all the plasmonic nanoparticles in this study present plasmon excitations with energies wellabove this threshold, their relative energies are not supposed to play a major role in the electron injection mechanism, in contrast to what has been previously postulated. ${ }^{23}$

Since the generation of hot electrons depends on the shape of plasmonic nanoparticles, the extinction spectra of the three morphologies included in this study have been calculated with the COMSOL software. The experimental UV-visible-NIR spectra of the different-shaped AuNPs before and after their integration into the final composites does not lead to a noticeable change in the positions of their original plasmon resonances (Figure 2), indicating that the averaged dielectric function of a matrix medium for the composites can be considered almost unaffected. Thus, the matrix dielectric function close to the optical constant of water $\left(\varepsilon_{\mathrm{w}}=1.8\right)$ can be used while the dielectric function of gold is taken from the literature. ${ }^{37}$ For the matrix dielectric constant, the following parameters have been used: $\varepsilon_{0}=2.2,2.1$ and 2.0 for NSs, NRs and NSTs, respectively. The slightly different matrix constants for different nanocrystals have been introduced in order to reproduce small plasmon shifts between the free AuNPs and the $\mathrm{SiO}_{2} @ \mathrm{AuNP} @ \mathrm{TiO}_{2}$ complexes (Figure 2). The dimensions of the AuNPs (NSs, NRs and NSTs) were taken according to the microcopy data (Figure 1 and Supporting Information). Therefore, the number of photo- 
excited electrons per unit of energy in the flat interface between the metal and the semiconductor is given by the normal component of the electric field at the surface: ${ }^{16,33}$

$$
\frac{d n}{d E}=A \cdot d s \cdot \frac{\left|E_{n o r m a l}\right|^{2}}{(\hbar \omega)^{4}}
$$

where ds is the element of surface of a nanoparticle, the constant A includes the material parameters of gold, ${ }^{16,33}$ the factor $\omega^{-4}$ comes from the quantum amplitudes of optical transitions in the Fermi gas of AuNPs and the normal electric field $E_{\text {normal }}$ should be calculated near this surface element. Then, the total number of high-energy electrons in a nanoparticle per unit energy should be calculated as a surface integral:

$$
\frac{d N(\lambda)}{d E}=\frac{A}{(\hbar \omega)^{4}} \int\left|E_{\text {normal }}\right|^{2} d s
$$

Since AuNPs are randomly oriented in solution, all orientations should be taken into consideration through an averaging process when anisotropic objects (NRs and NSTs) are studied. Following this model, the efficiencies for the generation of energetic electrons as a function of the wavelength of light for the three morphologies of AuNPs (nanospheres, nanostars and nanorods) are represented in Figure 5c. The positions of the maxima reproduce correctly those of the plasmon bands obtained experimentally. Nevertheless, the experimental plasmonic signatures are broader compared to those obtained from theory due to the fact that the experimental system is comprised by a dispersion of nanoparticles with different sizes. On the other hand, when the optical response is simulated, a monodisperse system is evaluated since the morphology of the nanoparticles is obtained from the averaged experimental dimensions. 

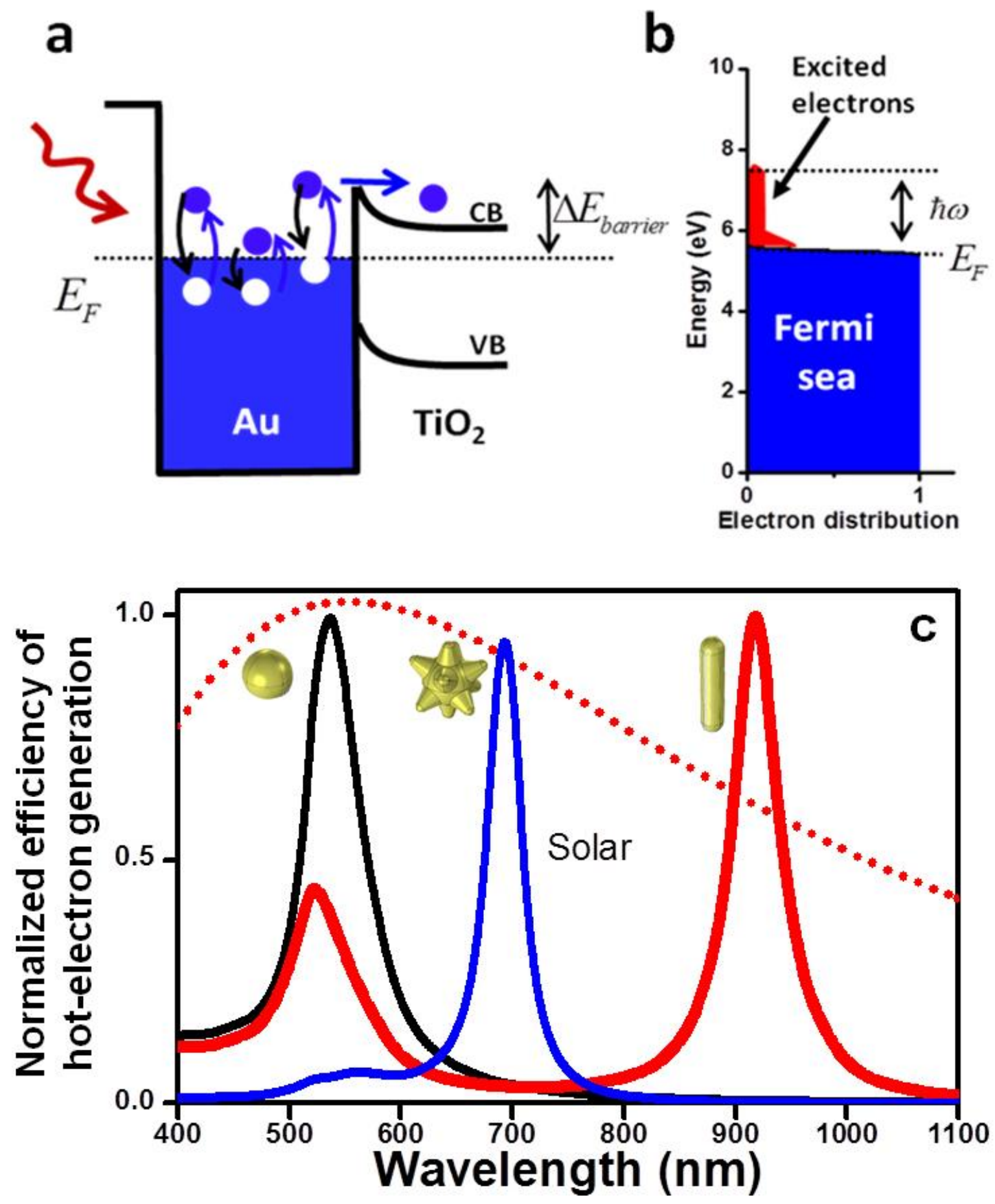

Figure 5. (a) Illustration of the excitation and relaxation processes in the Fermi sea of electrons of a gold nanoparticle and the subsequent transfer of a certain population of these excited electrons through the Schottky barrier between the gold nanoparticle and titania; (b) Equilibrium distribution of electrons (blue) and the photoexcited non-equilibrium contribution (red); (c) Spectral functions for the calculated rates of generation of highenergy electrons for the three morphologies of AuNPs: nanosphere (black), nanostar (blue), nanorod (red). The NST spectrum is given for the spike length of $10.7 \mathrm{~nm}$. The matrix dielectric function was taken as $\varepsilon_{0}=2.1$. The dotted red line represents the normalized spectrum of solar radiation. 
Once the optical excitations of the metal nanoparticles have been simulated, the rate of hot-electron injection can be studied. The experiment involves the solar-like spectrum in the interval between 350 and $2400 \mathrm{~nm}$. Therefore, assuming that the energy barrier is $\sim 1 \mathrm{eV}$, the integration should be performed over the interval between 350 and $1240 \mathrm{~nm}$. Since the main contribution to the rate of the photoreaction comes from the plasmon peaks, which are in the visible and near-IR regions of the electromagnetic spectrum, the exact number for the upper limit of the wavelength is not essential. Then, the total rate for the electron transfer from the metal nanoparticles to the semiconductor can be estimated as:

$$
\begin{aligned}
& \text { Rate }_{\text {transfer }}=\text { const } \cdot \frac{I_{0}}{V_{N P}} \int_{1239}^{350} \mathrm{~nm} n \times\left(\frac{h c}{\lambda}-\Delta E_{\text {barrier }}\right) \times \frac{d N(\lambda)}{d E} \cdot I_{\text {solar }}(\lambda) \text {, } \\
& I_{\text {solar }}(\lambda)=\frac{\text { const }^{\prime}}{\lambda^{5}} \frac{1}{e^{\frac{h c}{\lambda \cdot k T_{\text {sun }}}}-1} \\
& T_{\text {sun }}=5250 \mathrm{~K} \text {. }
\end{aligned}
$$

Herein, $\mathrm{I}_{0}$ and $\mathrm{dI}$ solar $(\lambda) / \mathrm{d} \lambda$ are the incident intensity and the solar spectrum, respectively, and $\mathrm{V}_{\mathrm{NP}}$ is the volume of the metal nanoparticle. The constant in the expression for Rate $e_{\text {transfer }}$ depends on the specifics of electron transfer from a metal nanoparticle to a semiconductor. Moreover, the rate presented above is divided by the volume of the nanoparticle, since the different samples studied in this work are endowed with the same number of metal atoms. Finally, the factors responsible for the generation of high-energy electrons that participate in the photochemical reaction can be enumerated from the simplified equation of the transfer rate:

Rate $_{\text {transfer }} \propto I_{0} \cdot\left(S_{N P} / V_{N P}\right) \cdot I_{\text {solar }}\left(\lambda_{\text {plasmon }}\right) \cdot \lambda_{\text {plasmon }}^{4} \cdot \operatorname{Enh}\left(\lambda_{\text {plasmon }}\right) \cdot \Delta \lambda_{\text {plasmon peak }}(2)$

where $\lambda_{\text {plasmon }}$ is the plasmon wavelength and $S_{N P}$ is the total surface area of the metal nanoparticle. To derive (2), the assumption that the plasmon peak is the dominant contribution of the optical spectrum must be taken. Therefore (2) shows that the rate of the plasmon-driven electron transfer depends on four main factors: net surface 
area, plasmonic enhancement factor, plasmon wavelength and intensity of the generation of high-energy electrons

(Figure 6a). Moreover, the enhancement factor presented above is given by:

$$
\operatorname{Enh}(\lambda)=\frac{1}{s_{N P}} \int\left|\frac{E_{\text {normal }}}{E_{0}}\right|^{2} d s
$$

where $\mathrm{E}_{0}$ is the incident electric field.

The average reaction rates of the photodegradation reactions performed in the presence of the different nanohybrids are evaluated as Rate experimental $=\Delta \mathrm{Absorbance} / \Delta \mathrm{t}$, where the time interval is $\Delta \mathrm{t}=200$ minutes. For convenience, all rates are normalized with respect to the NS rate. In this manner, when the reaction rates of photodegradation for the three different morphologies of AuNPs are compared, the following sequence is obtained: $\mathrm{R}_{\mathrm{NS}}<\mathrm{R}_{\mathrm{NR}}<\mathrm{R}_{\mathrm{NST}}$. In the same way, the relative rates with respect to the case of the nanospheres are: $r_{N S}=1, r_{N R}=R_{N R} / R_{N S}, r_{N S T}=R_{N S T} / R_{N S}$. The measured relative reaction rates for the photodegradation of $R h B$ and those calculated for the hot electron injection of the given nanoparticles obtained following the equations presented above are represented together in Figure 6e. This representation shows that the averaged rate of the $\mathrm{SiO}_{2} @ \mathrm{AuNR} @ \mathrm{TiO}_{2}$ for the decomposition of RhB is just slightly larger than that of the $\mathrm{SiO}_{2} @ \mathrm{AuNS} @ \mathrm{TiO}_{2}$. Moreover, simulations show a quite similar trend since NRs and NSs give rise to very close reaction rates for hotelectron injection. At this point, it is important to remind that even though AuNRs present strong plasmon enhancement when the incident electromagnetic field is parallel to the NR axis, the random distribution of these objects in solution (or adsorbed onto the $\mathrm{SiO}_{2}$ beads), leads to a reduction in the net enhancement (Figure 6d). This factor, together with the small surface-to-volume ratio of AuNRs and the fact that the maximum of the solar spectrum $(\sim 500 \mathrm{~nm})$ is close to the absorption maxima of the AuNSs $(520 \mathrm{~nm})$ may lead to the similar performance of NRs and NSs observed in this study (Figure 5d). As previously shown in Figure 3c, when $\mathrm{SiO}_{2} @ \mathrm{AuNST} @ \mathrm{TiO}_{2}$ is used as photocatalyst a 100\% increase in the reaction rate is observed when compared with the other two samples. Theoretical results show the same tendency as a result of the large plasmonic enhancement factors obtained in AuNSTs due to the increased field concentration at the spikes (Figure 6a-d). It is important to remark that in the particular case of the AuNSTs the theoretical calculation for the rate of hotelectron transfer is extremely sensitive to the morphology of the plasmonic component. In this vein, calculations 
show that the plasmonic enhancement depends strongly on the rounding radius and length of the spikes. Therefore, in the particular case of the AuNSTs only qualitative agreement between theory and experiment can be attained. In this regard, when simulations are performed for AuNSTs in which the length of the spikes are tuned between 6 and $12.6 \mathrm{~nm}$, an even larger enhancement in the reaction rate is obtained (red triangles in the right side of Figure 6e). It is also essential to remark that the specific surface area of each plasmonic component does not play an important role on their respective photocatalytic activities since their values do not vary substantially from one morphology to another (Figure S8). Overall, quantitative agreement between experiment and theory is obtained since the hot-electron injection mechanism results in a feasible explanation for the experimental observations. 
a $\quad \mathrm{NS}, \mathrm{E} \| \mathrm{z}, \lambda_{0}=520 \mathrm{~nm}$

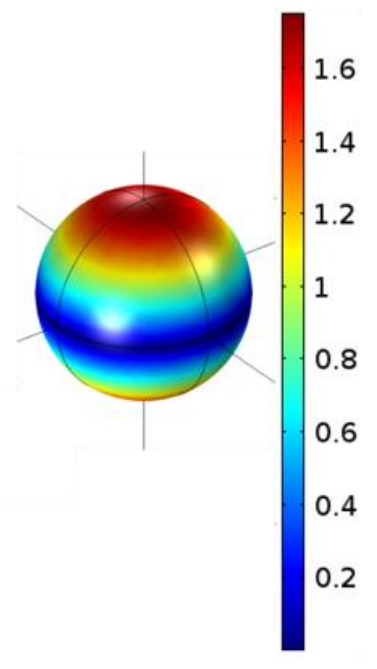

Diameter $=14 \mathrm{~nm}$ b

$\mathrm{NR}, \mathrm{E} \| \mathrm{z}, \lambda_{0}=925 \mathrm{~nm}$

C NST, E ||$z, \lambda_{0}=720 \mathrm{~nm}$
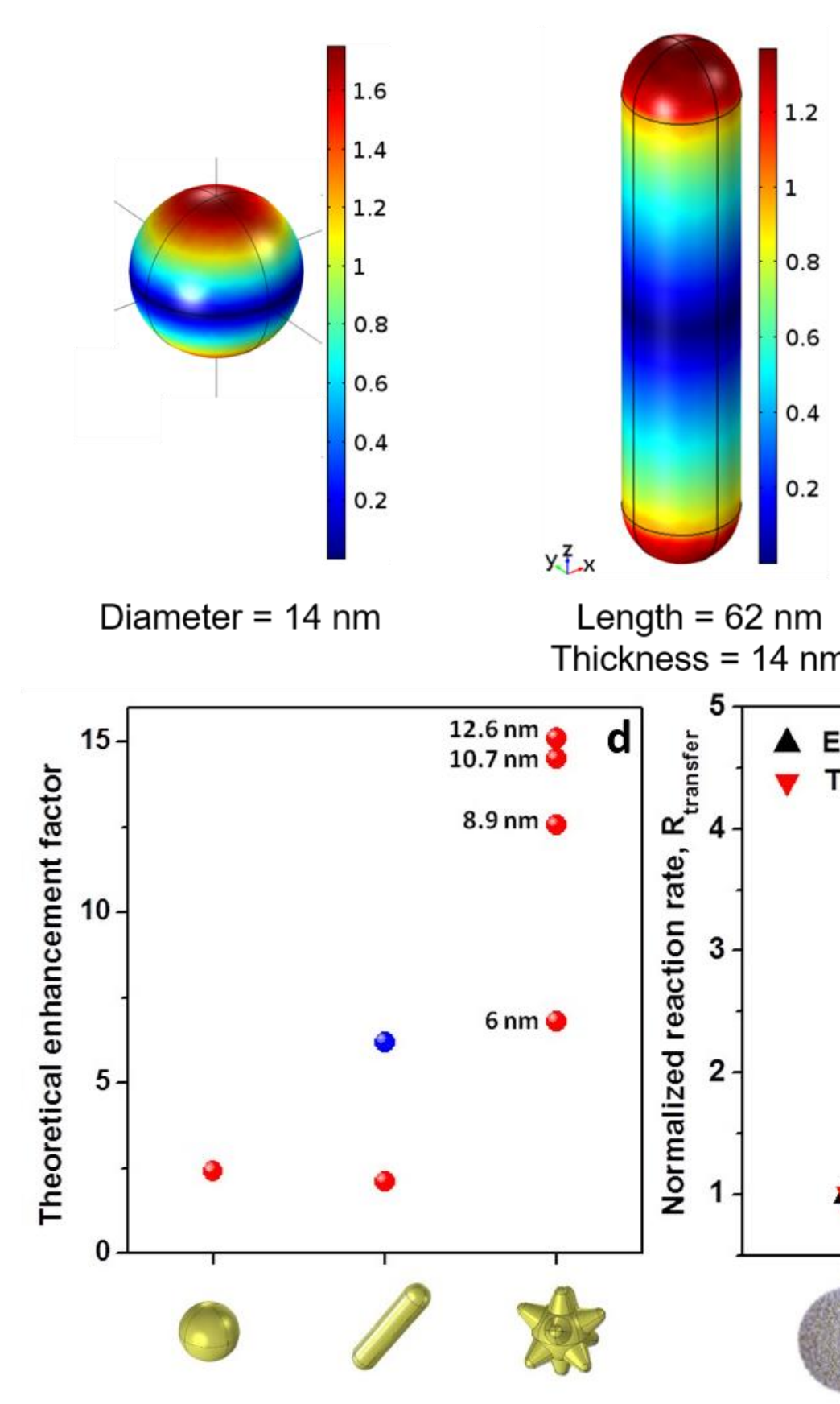

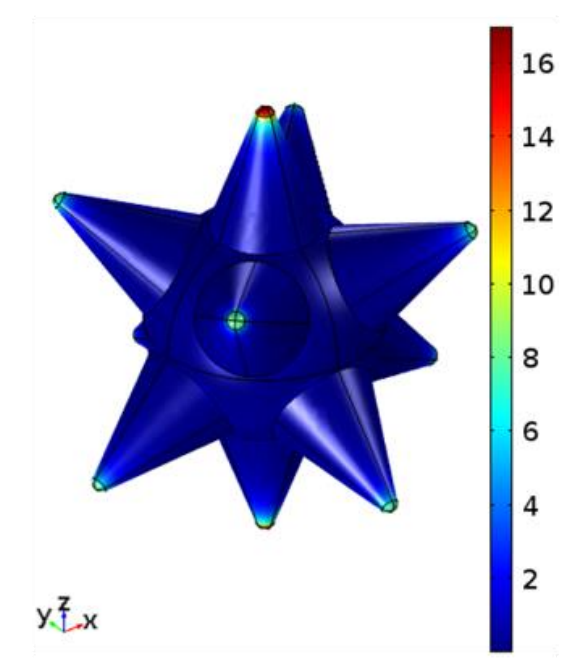

Core diameter $=25 \mathrm{~nm}$ Spike length $=12.6 \mathrm{~nm}$

Figure 6. (a-c) Calculated normal-to-surface field maps for the NSs, NRs and NSTs. The maps are computed for the plasmon-peak wavelengths. (d) Theoretical enhancement factors for the three different morphologies. In the case of the AuNST, the theoretical enhancement factor has been calculated for a core of $25 \mathrm{~nm}$ and different spike lengths. The blue dot corresponds to the enhancement factor of the AuNR when the object is parallel to the incident electric field vector; (e) Normalized reaction rates derived from the experiments (black triangles) and theory (red triangles) for the three different samples. The theoretical hot electron injection has been also calculated as a function of the length of the spikes in the AuNSTs. 
In summary, $\mathrm{SiO}_{2} @ \mathrm{AuNP} @ \mathrm{TiO}_{2}$ nanocomposites presenting a remarkable stability and dispersibility have been successfully fabricated and applied to the degradation of a model organic pollutant. In this case, both experiments and simulations show that the catalytic efficiency of these architectures is strongly dependent on the anisotropy of the plasmonic component. This is due to the different degree of electromagnetic field enhancement attained in each case. Among the different morphologies evaluated, the AuNST-containing nanocomposites show the highest photocatalytic activity, which is due to the intense local fields around the spikes of these nano-objects. This effect together with the strong scattering of the incident photons lead to a more efficient transfer of hot electrons to the semiconductor and the subsequent formation of electron-hole pairs that participate in the formation of the necessary radical species that promote the degradation of the pollutant. The obtained results show the critical influence of the anisotropy of the plasmonic component and open the door to a more rational design of photocatalytic nanoreactors capable of operating in the visible and NIR region of the electromagnetic spectrum.

\section{EXPERIMENTAL METHODS}

Materials: Tetraethylorthosilicate $98 \%$ (TEOS), ammonium hydroxide solution $28-30 \% \quad \% \quad\left(\mathrm{NH}_{4} \mathrm{OH}\right)$, poly(allylamine hydrochloride) (PAH, MW: 17500), sodium chloride (NaCl), tetrachloroauric acid $\left(\mathrm{HAuCl}_{4} \cdot 3 \mathrm{H}_{2} \mathrm{O}\right)$, Tetrakis(hydroxymethyl)phosphonium chloride solution (THPC), sodium hydroxide $(\mathrm{NaOH})$, sodium citrate $\left(\mathrm{Na}_{3} \mathrm{C}_{6} \mathrm{H}_{5} \mathrm{O}_{7}\right)$, silver nitrate $\left(\mathrm{AgNO}_{3}\right)$, sodium borohydride $\left(\mathrm{NaBH}_{4}\right)$, L- ascorbic acid $\left(\mathrm{C}_{6} \mathrm{H}_{8} \mathrm{O}_{6}\right)$, concentrated hydrochloric acid $(\mathrm{HCl})$, cetyltrimethyl ammonium bromide (CTAB), poly(vinylpyrrolidone) (PVP, MW: 10000), poly(styrene sulphonate) (PSS, MW: 14900) and N,N-dimethylformamide anhydrous (99,8 \%) $\left(\mathrm{HCON}\left(\mathrm{CH}_{3}\right)_{2}\right)$ were purchased from Sigma-Aldrich. $\mathrm{TiO}_{2}$ nanoparticles $5 \mathrm{~nm}$ (anatase $\geq 99 \%$ ) were purchased from Nanoamor. All chemicals were uses as received. Pure grade ethanol and Milli-Q grade water were used in all preparations.

Synthesis and fuctionalization of silica beads: Monodisperse silica spheres $(504 \pm 20 \mathrm{~nm})$ were prepared using a modified Stöber method. ${ }^{27}$ Typically, a TEOS solution $(1.7 \mathrm{~mL}, 1.2 \mathrm{M})$ was added to a solution containing ethanol $(18.12 \mathrm{~mL})$, ammonium hydroxide $(1.96 \mathrm{~mL})$, and water $(3.21 \mathrm{~mL})$. This mixture was stirred at room temperature for $2 \mathrm{~h}$. The excess of reagents was removed by three centrifugation-redispersion cycles with ethanol 
(6000 rpm, $20 \mathrm{~min}$ ). Subsequently, the silica beads were functionalized with poly(allylamine hydrochloride). To this end, $\mathrm{PAH}$ was dissolved in $0.5 \mathrm{M} \mathrm{NaCl}(\mathrm{pH} 5.0)$ with a final polymer concentration of $1 \mathrm{mg} / \mathrm{mL}$. Then, 25 $\mathrm{mL}$ of the positively charged PAH solution were added to the silica nanoparticles $(20 \mathrm{mg})$ and stirred at room temperature for $20 \mathrm{~min}$. The excess of reagents was removed by three centrifugation-redispersion cycles with water (6000 rpm, $20 \mathrm{~min})$.

Synthesis of gold nanospheres (AuNSs): $2.6 \mathrm{~nm}$ gold nanospheres were prepared as described elsewhere. ${ }^{38}$ A stable dispersion of $2.60 \pm 0.64 \mathrm{~nm}$ AuNSs was attained. $14 \mathrm{~nm}$ AuNSs were prepared by the classical method described by Turkevich, ${ }^{39}$ obtaining nanoparticles with a diameter of $13.8 \pm 1.47 \mathrm{~nm}$.

Synthesis of gold nanostars (AuNSTs): Gold nanostars were synthesized as described elsewhere. ${ }^{40}$ This protocol was further extended to enable size control of the stars with a core diameter of $25 \pm 5 \mathrm{~nm}$ and spike length ranging from 6 to $15 \mathrm{~nm}$ using a [HAuCl 4$]:[\mathrm{Au}$ seed] ratio of 11.25 .

Synthesis of gold nanorods (AuNRs) and polyelectrolyte coating: AuNRs were synthesized as described elsewhere. ${ }^{41}$ The dimensions obtained from TEM were $65 \pm 4 \mathrm{~nm}$ of length and $13 \pm 1 \mathrm{~nm}$ of thickness. The AuNRs were subsequently coated with a layer of a negatively charged polyelectrolyte (PSS) in order to proceed to the deposition onto the positively charged silica beads. ${ }^{42}$ In addition, and for comparison purposes, AuNRs with different aspect ratios: $62 \mathrm{~nm}$ x $20 \mathrm{~nm}$ (short AuNRs) and 105 x $16 \mathrm{~nm}$ (long AuNRs) were synthesized following the work by Ye and coworkers with slight modifications. ${ }^{43}$

Deposition of AuNSs, NSTs and NRs onto functionalized silica beads: A solution of nanoparticles $(2.25 \mathrm{~mL}$ of $14 \mathrm{~nm}$ AuNSs $5 \cdot 10^{-4} \mathrm{M}, 1.125 \mathrm{~mL}$ of $2.6 \mathrm{~nm}$ AuNSs $1 \cdot 10^{-3} \mathrm{M}, 2.55 \mathrm{~mL}$ of AuNSTs $4.41 \cdot 10^{-4} \mathrm{M}$ or $0.1664 \mathrm{~mL}$ of AuNRs $6.76 \cdot 10^{-3} \mathrm{M}$ ) were added to $20 \mathrm{mg}$ of functionalized silica nanoparticles. The mixture was stirred at room temperature for $20 \mathrm{~min}$. In all cases, the excess of AuNPs was removed by three centrifugation-redispersion cycles with water (3000 rpm, $20 \mathrm{~min})$. Finally, the product was redispersed in water $(10 \mathrm{~mL})$. 
Funcionalization of $\mathrm{SiO}_{2} @ \mathrm{Au}$ : $20 \mathrm{mg}$ of $\mathrm{SiO}_{2} @ \mathrm{Au}$ in $10 \mathrm{~mL} \mathrm{H} \mathrm{H}_{2} \mathrm{O}$ were funtionalized with $25 \mathrm{~mL}$ of the PAH solution described above. The excess of reagents was removed by three centrifugation-redispersion cycles with water $(3500 \mathrm{rpm}, 20 \mathrm{~min})$. Finally, the product was redispersed in water $(10 \mathrm{~mL})$.

Deposition of $\mathrm{TiO}_{2}: 20 \mathrm{mg}$ of $\mathrm{TiO}_{2}(5 \mathrm{~nm})$ redispersed in $100 \mathrm{~mL}$ of a sodium citrate solution $\left(1 \cdot 10^{-3} \mathrm{M}\right)$ were sonicated for $1 \mathrm{~h}$ with an ultrasonic tip. The excess of sedimented $\mathrm{TiO}_{2}$ was removed by centrifugation $(3500$ rpm, $10 \mathrm{~min})$. Then, $10 \mathrm{~mL}$ of $\mathrm{SiO}_{2} @ \mathrm{Au}(2 \mathrm{mg} / \mathrm{mL})$ were added to the solution of $\mathrm{TiO}_{2}$ and stirred at room temperature for $90 \mathrm{~min}$. The excess of $\mathrm{TiO}_{2}$ was removed by three centrifugation-redispersion cycles with water (3000 rpm, $20 \mathrm{~min})$. Finally, the product was redispersed in water $(10 \mathrm{~mL})$. The ratio $\mathrm{Au} / \mathrm{TiO}_{2}$ is $12.28 \% \mathrm{wt}$.

Photocatalytic study: The photocatalytic activity of the $\mathrm{SiO}_{2} @ \mathrm{Au} @ \mathrm{TiO}_{2}$ composites was evaluated by the degradation of Rhodamine $\mathrm{B}(\mathrm{RhB})$ in an aqueous solution under light irradiation from a $300 \mathrm{~W}$ Xe lamp with a cutoff filter to block a portion of UV light (LOT solar simulator 350-2400nm). $20 \mathrm{mg}$ of the hybrid catalysts were dispersed in $10 \mathrm{~mL}$ of Milli-Q water by using ultrasonication for $10 \mathrm{~min}$. Then, these photocatalysts were added to an aqueous solution of $\mathrm{RhB}\left(1 \times 10^{-5} \mathrm{M}\right)$. The mixture was stirred for $1 \mathrm{~h}$ in the dark to blend well and allow the adsorption-desorption equilibrium before the irradiation. The suspension was magnetically stirred at $\mathrm{pH} 5$ and the temperature of the solution was kept stable at $25^{\circ} \mathrm{C}$ inside a digitally controlled water bath. Aliquots of $4 \mathrm{~mL}$ were taken with intervals of 30 min during the experiments in order to measure the variation in the absorbance. When necessary, a glass colored filter that blocks radiation with wavelengths below $700 \mathrm{~nm}$ was introduced in the solar simulator.

Characterization: TEM images were obtained using a JEOL JEM 1010 transmission electron microscope operating at an acceleration voltage of $100 \mathrm{kV}$. HRTEM and elemental mapping by XEDS analysis were carried out with a JEOL JEM 2010F transmission electron microscope operating at an acceleration voltage of $200 \mathrm{kV}$. SEM images were obtained using SEM FEG JEOL JSM 6700F scanning electron microscope. UV-Vis spectra were obtained with Hewlett Packard HP8453 and Cary 5000 spectrophotometers.

\section{SUPPORTING INFORMATION}


Additional electron microscopy images of the nanohybrids and photodegradation profiles of RhB, EDX spectra, and calculations for the surface-to-volume ratios of the three models used in the present work. This information is available free of charge via the Internet at http://pubs.acs.org.

\section{AUTHOR INFORMATION}

Corresponding authors:

Miguel A. Correa-Duarte (E-Mail: macorrea@uvigo.es). Phone: +34 986813810

Alexander O. Govorov (E-mail: govorov@ helios.phy.ohiou.edu).

\section{NOTES}

The authors declare no competing financial interest.

\section{ACKNOWLEDGMENTS}

This work was funded by Xunta de Galicia (INBIOMED-FEDER "unha maneira de facer Europa" and EM2014/035), MINECO-Spain (CTM2014-58481-R), Fundación Tatiana Pérez de Guzmán el Bueno, and European Union Seventh Framework Programme [FP7/ REGPOT-2012-2013.1] under Grant Agreement no. 316265, BIOCAPS. A.O.G. was supported by the Army Office of Research (MURI Grant W911NF-12-1-0407) and by the Volkswagen Foundation. X.-T. K. was supported by IFFS/UESTC oversea postdoc program and Changjiang Scholar funding.

\section{REFERENCES}

(1) Fujishima, A.; Zhang, X.; Tryk, D. A. TiO 2 Photocatalysis and Related Surface Phenomena. Surf. Sci. Rep. 2008, 63, 515-582.

(2) Chen, X.; Selloni, A. Introduction: Titanium Dioxide $\left(\mathrm{TiO}_{2}\right)$ Nanomaterials. Chem. Rev. 2014, 114, $9281-$ 9282.

(3) Zhang, G.; Kim, G.; Choi, W. Visible Light Driven Photocatalysis Mediated via Ligand-to-Metal Charge Transfer (LMCT): An Alternative Approach to Solar Activation of Titania. Energy Environ. Sci. 2014, 7, 954-966.

(4) Devi, L. G.; Kavitha, R. A Review on Non Metal Ion Doped Titania for the Photocatalytic Degradation of Organic Pollutants under UV/Solar Light: Role of Photogenerated Charge Carrier Dynamics in Enhancing the Activity. Appl. Catal. B Environ. 2013, 140-141, 559-587.

(5) Dozzi, M. V.; Selli, E. Doping $\mathrm{TiO}_{2}$ with P-Block Elements: Effects on Photocatalytic Activity. $J$. Photochem. Photobiol. C-Photochem. Rev. 2013, 14, 13-28.

(6) Xu, H.; Ouyang, S.; Liu, L.; Reunchan, P.; Umezawa, N.; Ye, J. Recent Advances in TiO $2-\mathrm{Based}$ Photocatalysis. J. Mater. Chem. A 2014, 2, 12642-12661.

(7) Primo, A.; Corma, A.; García, H. Titania Supported Gold Nanoparticles as Photocatalyst. Phys. Chem. 
Chem. Phys. 2011, 13, 886-910.

(8) Ingram, D. B.; Christopher, P.; Bauer, J. L.; Linic, S. Predictive Model for the Design of Plasmonic Metal/Semiconductor Composite Photocatalysts. ACS Catal. 2011, 1, 1441-1447.

(9) Cushing, S. K.; Li, J.; Meng, F.; Senty, T. R.; Suri, S.; Zhi, M.; Li, M.; Bristow, A. D.; Wu, N. Photocatalytic Activity Enhanced by Plasmonic Resonant Energy Transfer from Metal to Semiconductor. J. Am. Chem. Soc. 2012, 134, 15033-15041.

(10) Cushing, S. K.; Li, J.; Bright, J.; Yost, B. T.; Zheng, P.; Bristow, A. D.; Wu, N. Controlling PlasmonInduced Resonance Energy Transfer and Hot Electron Injection Processes in Metal@TiO 2 Core-Shell Nanoparticles. J. Phys. Chem. C 2015, 119, 16239-16244.

(11) Li, J.; Cushing, S. K.; Meng, F.; Senty, T. R.; Bristow, A. D.; Wu, N. Plasmon-Induced Resonance Energy Transfer for Solar Energy Conversion. Nat. Photonics 2015, 9, 601-607.

(12) Mubeen, S.; Lee, J.; Singh, N.; Krämer, S.; Stucky, G. D.; Moskovits, M. An Autonomous Photosynthetic Device in Which All Charge Carriers Derive from Surface Plasmons. Nat. Nanotechnol. 2013, 8, $247-251$.

(13) Govorov, A. O.; Zhang, H.; Gun'ko, Y. K. Theory of Photoinjection of Hot Plasmonic Carriers from Metal Nanostructures into Semiconductors and Surface Molecules. J. Phys. Chem. C 2013, 117 (32), 1661616631.

(14) Furube, A.; Du, L.; Hara, K.; Katoh, R.; Tachiya, M. Ultrafast Plasmon-Induced Electron Transfer from Gold Nanodots into $\mathrm{TiO}_{2}$ Nanoparticles. J. Am. Chem. Soc. 2007, 129, 14852-14853.

(15) Christopher, P.; Xin, H.; Marimuthu, A.; Linic, S. Singular Characteristics and Unique Chemical Bond Activation Mechanisms of Photocatalytic Reactions on Plasmonic Nanostructures. Nat. Mater. 2012, 11, 1044-1050.

(16) Zhang, H.; Govorov, A. O. Optical Generation of Hot Plasmonic Carriers in Metal Nanocrystals: The Effects of Shape and Field Enhancement. J. Phys. Chem. C 2014, 118, 7606-7614.

(17) Harutyunyan, H.; Martinson, A. B. F.; Rosenmann, D.; Khorashad, L. K.; Besteiro, L. V; Govorov, A. O.; Wiederrecht, G. P. Anomalous Ultrafast Dynamics of Hot Plasmonic Electrons in Nanostructures with Hot Spots. Nat. Nanotechnol. 2015, 10, 770-774.

(18) Daniel, M. C.; Astruc, D. Gold Nanoparticles: Assembly, Supramolecular Chemistry, Quantum-SizeRelated Properties, and Applications Toward Biology, Catalysis, and Nanotechnology. Chem. Rev. 2004, 104, 293-346.

(19) Liz-Marzán, L. M. Tailoring Surface Plasmons through the Morphology and Assembly of Metal Nanoparticles. Langmuir 2006, 22, 32-41.

(20) Kochuveedu, S.; Kim, D. D. Surface-Plasmon-Induced Visible Light Photocatalytic Activity of $\mathrm{TiO}_{2}$ Nanospheres Decorated by Au Nanoparticles with Controlled Configuration. J. Phys. Chem. C 2012, 116, 2500-2506.

(21) Oros-Ruiz, S.; Pedraza-Avella, J. A.; Guzmán, C.; Quintana, M.; Moctezuma, E.; del Angel, G.; Gómez, R.; Pérez, E. Effect of Gold Particle Size and Deposition Method on the Photodegradation of 4Chlorophenol by $\mathrm{Au} / \mathrm{TiO}_{2}$. Top. Catal. 2011, 54, 519-526.

(22) Murdoch, M.; Waterhouse, G. I. N.; Nadeem, M. A.; Metson, J. B.; Keane, M. A.; Howe, R. F.; Llorca, J.; Idriss, H. The Effect of Gold Loading and Particle Size on Photocatalytic Hydrogen Production from Ethanol over $\mathrm{Au} / \mathrm{TiO}_{2}$ Nanoparticles. Nat. Chem. 2011, 3, 489-492.

(23) Li, A.; Zhang, P.; Chang, X.; Cai, W.; Wang, T.; Gong, J. Gold Nanorod@TiO 2 Yolk-Shell Nanostructures for Visible-Light-Driven Photocatalytic Oxidation of Benzyl Alcohol. Small 2015, 11, 1892-1899.

Hu, C.; Zhang, X.; Li, X.; Yan, Y.; Xi, G.; Yang, H.; Bai, H. Au Photosensitized TiO 2 Ultrathin Nanosheets 
with $\{001\}$ Exposed Facets. Chem. Eur. J. 2014, 20, 13557-13560.

(25) Kodiyath, R.; Manikandan, M.; Liu, L.; Ramesh, G. V; Koyasu, S.; Miyauchi, M.; Sakuma, Y.; Tanabe, T.; Gunji, T.; Duy Dao, T.; et al. Visible-Light Photodecomposition of Acetaldehyde by TiO2-Coated Gold Nanocages: Plasmon-Mediated Hot Electron Transport via Defect States. Chem. Commun. (Camb). 2014, $50(98), 15553-15556$.

(26) Alvarez-Puebla, R.; Liz-Marzán, L. M.; García de Abajo, F. J. Light Concentration at the Nanometer Scale. J. Phys. Chem. Lett. 2010, 1, 2428-2434.

(27) Stöber, W.; Fink, A.; Bohn, E. Controlled Growth of Monodisperse Silica Spheres in the Micron Size Range. J. Colloid Interface Sci. 1968, 26, 62-69.

(28) Sclafani, A.; Herrmann, J. M. Comparison of the Photoelectronic and Photocatalytic Activities of Various Anatase and Rutile Forms of Titania in Pure Liquid Organic Phases and in Aqueous Solutions. J. Phys. Chem. 1996, 100, 13655-13661.

(29) Xu, M.; Gao, Y.; Moreno, E. M.; Kunst, M.; Muhler, M.; Wang, Y.; Idriss, H.; Wöll, C. Photocatalytic Activity of Bulk $\mathrm{TiO}_{2}$ Anatase and Rutile Single Crystals Using Infrared Absorption Spectroscopy. Phys. Rev. Lett. 2011, 106, 138302.

(30) Bumajdad, A.; Madkour, M. Understanding the Superior Photocatalytic Activity of Noble Metals Modified Titania under UV and Visible Light Irradiation. Phys. Chem. Chem. Phys. 2014, 16, 7146-7158.

(31) Wu, J.; Yu, P.; Susha, A. S.; Sablon, K. A.; Chen, H.; Zhou, Z.; Li, H.; Ji, H.; Niu, X.; Govorov, A. O. et al. Broadband Efficiency Enhancement in Quantum Dot Solar Cells Coupled with Multispiked Plasmonic Nanostars. Nano Energy 2015, 13, 827-835.

(32) Wu, T.; Liu, G.; Zhao, J.; Hidaka, H.; Serpone, N. Photoassisted Degradation of Dye Pollutants. V. SelfPhotosensitized Oxidative Transformation of Rhodamine B under Visible Light Irradiation in Aqueous $\mathrm{TiO}_{2}$ Dispersions. J. Phys. Chem. B 1998, 102, 5845-5851.

(33) Govorov, A. O.; Zhang, H.; Demir, H. V.; Gun'ko, Y. K. Photogeneration of Hot Plasmonic Electrons with Metal Nanocrystals: Quantum Description and Potential Applications. Nano Today 2014, 9, 85-101.

(34) Govorov, A. O.; Zhang, H. Kinetic Density Functional Theory for Plasmonic Nanostructures: Breaking of the Plasmon Peak in the Quantum Regime and Generation of Hot Electrons. J. Phys. Chem. C 2015, 119, 6181-6194.

(35) Govorov, A. O.; Richardson, H. H. Generating Heat with Metal Nanoparticles. Nano Today 2007, 2, 3038.

(36) Li, W.; Coppens, Z. J.; Besteiro, L. V; Wang, W.; Govorov, A. O.; Valentine, J. Circularly Polarized Light Detection with Hot Electrons in Chiral Plasmonic Metamaterials. Nat. Commun. 2015, 6, 8379.

(37) Johnson, P. B.; Christy, R. W. Optical Constants of the Noble Metals. Phys. Rev. B 1972, 6, 4370-4379.

(38) Duff, D. G.; Baiker, A.; Edwards, P. P. A New Hydrosol of Gold Clusters. J. Chem. Soc. Chem. Commun. 1993, 272, 96.

(39) Turkevich, J.; Stevenson, P. C.; Hillier, J. A Study of the Nucleation and Growth Processes in the Synthesis of Colloidal Gold. Discuss. Faraday Soc. 1951, 11, 55-75.

(40) Kumar, P. S.; Pastoriza-Santos, I.; Rodriguez-Gonzalez, B.; García De Abajo, F. J.; Liz-Marzan, L. M. High-Yield Synthesis and Optical Response of Gold Nanostars. Nanotechnology 2008, 19, 015606.

(41) Scarabelli, L.; Sánchez-Iglesias, A.; Pérez-Juste, J.; Liz-Marzán, L. M. A “Tips and Tricks” Practical Guide to the Synthesis of Gold Nanorods. J. Phys. Chem. Lett. 2015, 6, 4270-4279.

(42) Pastoriza-Santos, I.; Pérez-Juste, J.; Liz-Marzán, L. M. Silica-Coating and Hydrophobation of CTABStabilized Gold Nanorods. Chem. Mater. 2006, 18, 2465-2467. 
(43) Ye, X.; Jin, L.; Caglayan, H.; Chen, J.; Xing, G.; Zheng, C.; Doan-Nguyen, V.; Kang, Y.; Engheta, N.; Kagan, C. R. et al. Improved Size-Tunable Synthesis of Monodisperse Gold Nanorods through the Use of Aromatic Additives. ACS Nano 2012, 6, 2804-2817. 


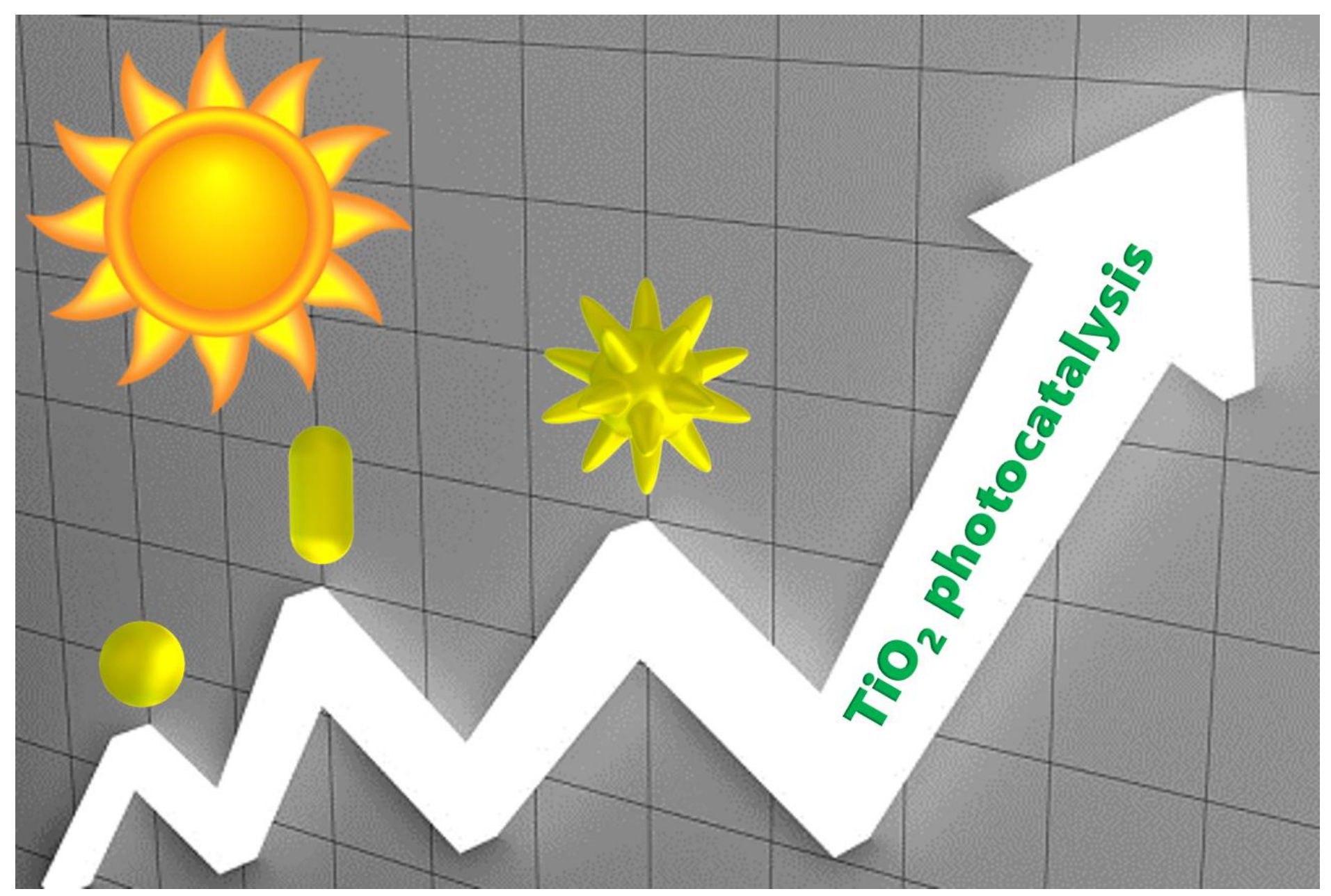

